\title{
Expertengespräch: Chronische Erkrankungen und Multimorbidität - Teil 2
}

Warum eignet sich die Ganzheits-

medizin besonders gut dazu,

chronische Erkrankungen zu

behandeln?

Prof. Dr. Dr. Bernhard Uehleke: Es ist ja vor allen Dingen die Naturheilkunde als Teil der Ganzheitsmedizin, die eine Anleitung zu einem gesunden, naturgemässen Leben gibt. Insofern betreibt man auf diese Weise Vorbeugung durch eine gesunde Lebensweise, die sich an der Natur orientiert. Die Naturheilkunde hilft, Zivilisationserkrankungen zu vermeiden oder, wenn sie bereits im Entstehen begriffen sind, sie noch aufzuhalten und $\mathrm{zu}$ verzögern. Das ist der genuine Ansatz der Naturheilkunde.

Was ist das Genuine daran, denn viele Methoden haben sich die Prävention von Krankheiten auf die Fahne geschrieben?

Es geht hier spezieller um Zivilisationskrankheiten und um die Zivilisation mit ihren Folgeerscheinungen: $\mathrm{zu}$ viel Stress, zu viel psychische Belastung, zu wenig körperliche Tätigkeit. Wir haben im modernen Leben eine Schieflage, die sich in stressbedingten Folgeerkrankungen in vielen Bereichen - wie Herz-Kreislauf-Erkrankungen, Psyche, bei manchen schlägt es auch auf Magen-DarmFunktionen, z.B. in Form von Reizma-

Wer gesund bleiben will, muss mit dem modernen Leben vorsichtig umgehen. Die eigene Autonomie ist wichtig, um psychische Belastungen zu vermeiden. Prof. Dr. Dr. Bernhard Uehleke rät zu einem naturgemässen Lebenswandel.

gen oder Reizdarm - manifestieren. Die natürliche Lebensweise versucht die Entstehung von Krankheiten zu beeinflussen. Es geht darum, einfach gesund zu leben, um z.B. durch einseitige Überernährung Stoffwechselerkrankungen zu vermeiden.

\section{Die Naturheilkunde beginnt mit der Lebensführung?}

Ja, aber sie geht wesentlich tiefer. Man muss sich fragen, wozu sich der Mensch im Laufe der Evolution entwickelt hat. Er ist so konstruiert, dass er sich ein bisschen bewegt und ansonsten mehr oder weniger seine Ruhe hat. Der Mensch ist auf keinen Fall dafür geschaffen, dass er den ganzen Tag in die Glotze schaut und sich mit schwierigen Gesprächen, die ihn ärgern, in Bedrängnis bringen und unter Stress setzen lässt.

\section{Das moderne Leben steht dem allerdings diametral entgegen.}

Das stimmt, und auf den ersten Blick sieht das nach einer Abkehr von der Moderne aus. Natürlich geht es nur zum Teil, die richtigen Konsequenzen daraus zu ziehen. Aber es ist wichtig, dass man ganz bewusst Sport, Gymnastik und Spaziergänge in seinen Tagesablauf einbaut. Das brauchte der frühere Mensch nicht, der ge- nügend im Garten und auf dem Feld gearbeitet hat. Wir müssen das zum Ausgleich nachholen.

Sie empfehlen gestressten Menschen, das in den Terminkalender einzutragen?

Unbedingt, man muss sich dafür auch Zeit freihalten. Die Ordnungstherapie, ein klassisches Naturverfahren, kennt die Themen Stressbekämpfung durch Zeitmanagement, durch Planung oder durch die Vorbereitung auf bestimmte Situationen. Wichtig ist, den Stress durch Angst, durch Unvorbereitetsein und durch das Gefühl, sich ausgeliefert zu fühlen, zu reduzieren. Dabei geht es um Autonomie und um die Selbstwahrnehmung der eigenen Bedürfnisse. Man muss lernen, sie zu erkennen.

Patienten, die an einem Burn-out leiden, scheinen oft einen Verlust der Autonomie zu haben, weil sie ständig unter Anspannung stehen.

Das ist die Selbstwahrnehmung, auch wenn sie objektiv betrachtet vielleicht gar nicht so unautonom sind. Aber sie fühlen sich unautonom, haben übertriebene Ängste vor der Zukunft oder mangelndes Selbstvertrauen. Wichtig ist, dass sie ihre Probleme relativieren. Dabei helfen mehr sozia- 


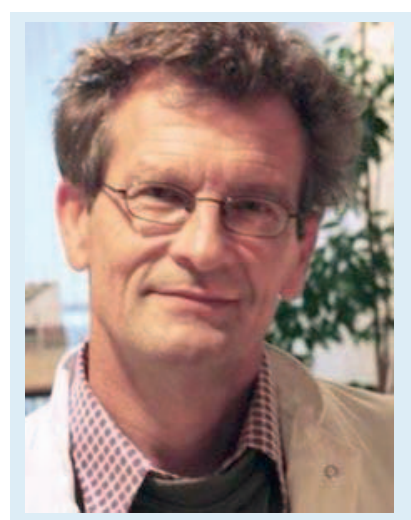

Prof. Dr. Dr. Bernhard Uehleke

(Bernhard.Uehleke@usz.ch) ist seit 2001 in der Abteilung Naturheilkunde der Charité in Berlin tätig und forscht am Immanuel Krankenhaus Berlin. Ausserdem arbeitet er am Institut für Naturheilkunde am Universitätsspital Zürich. Seit 2011 ist er Professor für Phytopharmakologie und Phytotherapie an der Hochschule für Gesundheit und Sport in Berlin.

"Kinder werden heute viel zu früh in die Erwachsenenrolle gedrängt, geraten über Internet und Facebook in Stress.»

«Die gute und wesentliche Arbeit muss auch mit weniger Zeitaufwand zu leisten sein.»

le Kontakte mit vernünftigen Leuten, dass man mal einen Philosophen liest oder vielleicht auch bei der Religion Trost und Rückhalt sucht. Freiräume sind nötig, und wenn man sich am Wochenende in den Garten setzt, kann man z.B. Finanzkrise Finanzkrise und Job Job sein lassen. Kleine Freiräume sind sehr wertvoll und stärken.

\section{Was empfehlen Sie konkret, wenn jemand aus dem mittleren Manage- ment bei Ihnen anklopft und fragt: «Ich fühle mich gestresst, was soll ich tun?»}

Ich weise immer sehr darauf hin, dass das Thema Stress, genauer Disstress, oft selbst gemacht und zweiseitig ist. Dass man einerseits zu grossen Ehrgeiz entwickelt und im sozialen Gefüge auf Äusserlichkeiten wie ein teures Auto, exotische Ferien, also auf Geld und materielle Güter und den damit verbundenen Status, grossen Wert legt. Man vergisst dabei, dass es im Leben noch anderes gibt. Oft hilft den Patienten die Frage: "Wirst $\mathrm{Du}$ in Deiner Sterbestunde sagen: «Ach, hätte ich doch noch mehr gearbeitet?> oder wirst $\mathrm{Du}$ vielleicht sagen: «Ach, hätte ich mich mal zurückgezogen, ausgeruht oder mich mal um meine Kinder gekümmert.»»

Um diese Einsicht umzusetzen, braucht es oft eine Art von Therapie, ein Programm oder ein Coaching. Dabei muss man auch die Fähigkeit der Patienten fördern, im Leben und im Beruf Prioritäten zu setzen. Man leistet nicht mehr, wenn man 60, 70 oder 80 Stunden arbeitet. Die gute und wesentliche Arbeit muss auch mit weniger Zeitaufwand zu leisten sein.

\section{Das bedeutet ein grosses Mass an Selbstreflexion bei Menschen, die sich zu viel aufladen?}

Es braucht tiefe psychologische Selbstreflexion, und man muss Partner und Leute haben, mit denen man reden und denen man vertrauen kann. Deshalb ist das Eingebundensein in soziale Netze auch ausserhalb der Arbeit ganz wichtig. Viele sind aber leider hauptsächlich an ihrem Arbeitsort mit anderen in Kontakt und sitzen abends allein und erschöpft vor dem Fernseher. In der Single-Gesellschaft ist da wirklich im wahrsten Sinne jeder mutterseelenallein. Diese Entwicklung müssen wir auch sehr kritisch hinterfragen. Familie und ein Gemeinschaftsleben brauchen wir für unsere Gesundheit. In dem Dorf, in dem ich lebe, gibt es jede Woche mehrfach eine Möglichkeit, um sich zu treffen und auch mit Gleichgesinnten schöne Gespräche führen. Im Endeffekt geht es um die Prioritäten: "Was will ich im Leben? Will ich alt und einsam und in der Managerstufe möglichst weit oben sterben oder will ich eine Familie haben, will ich Kinder und Enkel haben und nehme ich mir dafür die entsprechende Zeit?»

\section{Spielt bei den meisten chronischen}

Erkrankungen und Zivilisationserkrankungen Stress eine Rolle?

Bei vielen ist der Stress beteiligt. Die Stoffwechselerkrankungen wie Übergewicht und Hypercholesterinämie mit den Folgeerkrankungen sind ein Beispiel. Stress führt auch zu vermehrten Hungerattacken, und wenn man hier die Grundgegebenheiten einer genussvollen und langsamen Nahrungsaufnahme im sozialen Kontakt mit entsprechenden Gesprächen und langsamem Kauen und auch mal ein bisschen Reden über Bord wirft, schlingt man sich in einsamen Fressattacken den Bauch voll und kann seine Nahrungszufuhr und seine Kalorienaufnahme nicht regulieren. Ausserdem macht Stress hungrig.

Wichtig ist, dass Essen wieder seinen alten Stellenwert bekommt, dass man sich Zeit lässt. Es muss ordentlich gekocht sein, die Qualität muss stimmen und nicht Fastfood der billigsten Sorte sein, und vom Essen «on-the-go» müssen wir erst gar nicht reden.

\section{Wie ist die Reaktion von Eltern übergewichtiger Kinder auf diese Empfehlungen?}

Das sehen sie natürlich ein; die Umsetzung ist ja das Schwierige. Man muss Brücken bauen und in kleinen Schritten zum Ziel kommen. Bei Kindern ist das Verändern von Essverhalten ein besonderes Problem, weil es vererbt und gelernt wird. Die meisten Kinder, die übergewichtig sind, haben übergewichtige Eltern. Die Ernährungsmediziner und Ökotrophologen haben ja verschiedene Zugänge, um das Verhalten zu ändern. Es ist hilfreich zu lernen, dass der Körper auch eine Zeit lang ohne 
Nahrungsmittelaufnahme auskommt und man dann nicht gleich verhungert. Im Rahmen einer Fastenkur kann die Erkenntnis, jetzt gibt es nichts, ich lebe aber trotzdem und recht gut, einige Einsichten auslösen. Dieser Lerneffekt ist bei Kindern, aber auch bei Eltern wichtig, die glauben, wenn mal eine Mahlzeit ausfällt, bricht die Welt zusammen.

Die möglichen Ansätze muss man mit dem Patienten im Einzelnen besprechen, aber auch Diäten anbieten, bei denen man bestimmte Mahlzeiten auslässt oder auf Energieträger verzichtet, die reich an Zucker oder Fett sind. Es hilft, sich bewusst zu machen, dass Nahrung etwas sehr Differenziertes ist. Viele Methoden sind je nach Patient unterschiedlich erfolgreich. Man muss ihnen Verschiedenes anbieten.

Eignet sich die Naturheilkunde auch für die Behandlung von Zivilisationskrankheiten bei Kindern wie ADHS oder Neurodermitis?

Man muss sich einstimmend überlegen, wie anders heute das Leben von Kindern in unseren Ländern geworden ist, wenn man es mit dem vor 30 Jahren vergleicht. Kinder werden heute viel zu früh in die Erwachsenenrolle gedrängt, geraten über Internet und Facebook in Stress. Computerspiele lenken insbesondere Buben von ihren schulischen Aufgaben ab, dass sie in der Schule schwere Defizite haben. Das ist ein Phänomen, das sich durch alle Schichten zieht. Wenn sie weder von Erziehern noch von Eltern in den Griff zu bekommen sind, diagnostiziert man ADHS, das die Folge des Fehlens einer natürlichen Erziehung mit einer gewissen Autorität der Erwachsenen ist. Auch die neuen Medien haben eine Mitschuld, weil sie zum Bewegungsmangel beitragen und die Konzentrationsfähigkeit beeinträchtigen.

\section{Wie behandeln Sie in der Naturheilkunde die Neurodermitis?}

Es gibt Untersuchungen, die zeigen, dass der Neurodermitis eine Kommunikationsstörung ab dem Säuglingsalter zugrunde liegt, in dem das erste Signal zwischen Kind und Mut- ter, das Lächeln, ausgetauscht wird. Sehr vereinfacht ausgedrückt: Wenn die Mutter nicht lächelt, weil sie Stress hat, sucht das Kind nach Möglichkeiten, auf sich aufmerksam zu machen und eine andere Art der Kommunikation zu etablieren. Es kann zum Brüllkind werden, schreien und toben, sich in Richtung ADHS entwickeln, oder es kann anfangen, auf der Haut rumzukratzen. Auch wenn man den Eltern nicht immer die Schuld geben darf, zeigt dieser Zwang, sich zu kratzen und auf sich aufmerksam machen zu müssen, dass etwas grundsätzlich fehlgelaufen ist.

\section{Muss man diese Kommunikations- störung bei der Behandlung ansprechen?}

Das ist natürlich schwierig, weil man die Eltern sonst angreift. In der Praxis geht das nicht. Stattdessen muss man Mittelchen anbieten. Nachtkerzenöl innerlich und äusserlich und die Behandlung mit Heilerde, die man antrocknen lässt und auf diese Weise einfach auch der Haut mal andere Empfindungen anbietet.

Interview: Oliver Klaffke 